\title{
The Correlation Between SNOT-22 and Visual Analogue Scale in the Assessment of Symptom Severity in Chronic Rhinosinusitis
}

\author{
Omoregie Joseph Idugboe ${ }^{1, ~ *}$, Adebolajo Adeyemo ${ }^{2}$, Ebidisemene Linda Totyen ${ }^{1}$ \\ ${ }^{1}$ Department of Otorhinolaryngology, Federal Medical Center, Yenagoa, Nigeria \\ ${ }^{2}$ Department of Otorhinolaryngology, University College Hospital, Ibadan, Nigeria
}

Email address:

j_tookie@yahoo.com (O. J. Idugboe)

${ }^{*}$ Corresponding author

\section{To cite this article:}

Omoregie Joseph Idugboe, Adebolajo Adeyemo, Ebidisemene Linda Totyen. The Correlation Between SNOT-22 and Visual Analogue Scale in the Assessment of Symptom Severity in Chronic Rhinosinusitis. International Journal of Otorhinolaryngology.

Vol. 5, No. 1, 2019, pp. 24-30. doi: 10.11648/j.ijo.20190501.16

Received: May 25, 2019; Accepted: July 1, 2019; Published: July 13, 2019

\begin{abstract}
Chronic rhinosinusitis is an inflammatory process involving the sinonasal mucosa, lasting 12 weeks or longer. It is often characterized by a complex of debilitating symptoms usually classified into minor and major symptoms. Estimating symptom/ disease severity using structured questionnaires can be challenging. This is especially so in rural areas where a combination of low literacy levels and language barrier may present communication difficulties. In view of the aforementioned challenges, it has become imperative to explore the correlation between the 22- item sino-nasal outcome test (SNOT-22) and a simpler means of evaluating symptom severity in chronic rhinosinusitis; hence this study. To determine the correlation between SNOT-22 and visual analogue scale (VAS) questionnaire scoring of symptom severity in chronic rhinosinusitis. This was a twelve month prospective cross sectional study of fifty- two consecutive newly diagnosed adult patients with chronic rhinosinusitis. Age of patients ranged from $19-91$ years. Male to female ratio was 1: 1.26. There was a significant positive correlation between VAS and SNOT-22 in moderate and high symptom severity in patients with CRS. This study found a positive correlation between SNOT-22 and VAS scoring of disease severity in moderate and severe diseases and an insignificant correlation between these two instruments in mild disease. VAS can be used as a guide to symptom severity in the pre-treatment patients with CRS.
\end{abstract}

Keywords: Chronic Rhinosinusitis, Sinonasal Outcome Test-22, Visual Analogue Scale, Symptoms, Severity, Low Literacy, Language Barrier

\section{Introduction}

Chronic rhinosinusitis (CRS) is an inflammatory process involving the sinonasal mucosa. It is defined as the presence of two or more symptoms one of which should be either nasal blockage/obstruction/congestion or nasal discharge (anterior or posterior) with or without facial pain (pressure), hyposmia for more than 12 weeks, together with pathologic endoscopic findings in the middle nasal meatus and or computerized tomography scan changes within the osteomeatal complex (OMC) and or sinuses [1]. The mean medical cost of CRS per patient was reported to be $\$ 921$ with an average of 4.8 missed workdays annually in the United
States [2]. In north-western Nigeria, Iseh et al [3] reported a prevalence of $7.3 \%$ of CRS in a population of children and adults, which is consistent with studies on CRS in otolaryngology practice from various regions in Nigeria with a similar population [4-9].

Although the symptoms of rhinosinusitis are not life threatening, they are associated with a dramatic reduction in quality of life and causes emotional distress among sufferers. It often results to reduced work and school attendance, sleep disturbance, sexual and olfactory dysfunction [10]. These symptoms and signs of CRS which includes facial congestion or fullness, nasal obstruction or blockage, anterior rhinorhoea or post-nasal discharge, hyposmia, headache, fever, halitosis, 
fatigue, dental pain, cough and ear pressure or pain lasting are often troubling and refractive $[11,12]$.

Disease-specific questionnaires are frequently used to assess symptom severity in patients with CRS. Compared with general instruments they are able to capture symptoms in greater detail and are more sensitive in detecting changes after therapeutic intervention [13]. The severity of CRS symptoms often called a symptom score are measured using a disease specific health- related quality of life questionnaire [14-16].

One of the most validated instrument / questionnaire for evaluating disease severity and quality of life in CRS is the 22item Sinonasal Outcomes Test (SNOT-22). It combines the assessment of nasal, paranasal, and psychological symptoms, as well as those associated with sleep. It is a questionnaire which is broadly used in literature and is considered the most adequate questionnaire to assess the severity of CRS as well as the quality of life of patients with the disease [17]. It has 22 questions about sinonasal symptoms and general status aspects, rated on a 5 point scale (0-5) and has been used to grade CRS. A low/mild symptom severity equates to a SNOT-22 score of 0 to 26 (mild disease), an intermediate/moderate severity score to 27 to 58 (moderate disease) and high/severe symptom severity score equates to 59 and above (severe disease). Another one of such instrument that could be used in evaluation of CRS is the visual analogue scale questionnaire (VAS). The VAS is a validated psychometric response scale usually represented by a horizontal line, $10 \mathrm{~cm}$ in length, anchored by word descriptors at each end. The patient indicates the position on the line that they feel represents their perception of their current state [16]. Though it is a straightforward assessment tool widely employed in many disciplines to subjectively estimate the degree or strength of pain, its application in estimation of overall symptom severity in CRS is still vastly unexplored.

While it is true that structured self- administered questionnaires such as SNOT-22 has given patients the medium to finally express the level of debilitation or discomfort they suffer as a result of the disease, understanding the terminologies of some of these questionnaires can be challenging for some patients, especially in rural areas where literacy levels are low. Completion of these questionnaires usually takes a longer time, often requiring the assistance of medical personnel. This study aims to explore the correlation between SNOT-22 and a simpler, easy to administer alternative in VAS.

\section{Method}

This was a one year prospective cross-sectional study (April 2014 - March, 2015) of consecutive newly diagnosed adult patients with CRS presenting at the otorhinolaryngology clinic of Obafemi Awolowo University Teaching Hospitals complex, Ile-Ife, a tertiary institution in the south- west region of Nigeria.

Ethical clearance was sought and obtained for this work from the hospital Ethics review committee (Obafemi Awolowo University Teaching Hospital Complex IRB/IEC/0004553, Protocol number: ERC/2013/11/10) after which a written informed consent was obtained from individual participants in this study.

Exclusion criteria: Patients diagnosed with acute rhinosinusitis, asthma, depression as well as other chronic ailments (co-morbidities) were excluded from this study.

After a clinical and radiological confirmation of CRS, SNOT-22 questionnaire was administered to each patient upon their first clinical visit. This was to estimate the overall symptoms severity score and grade the disease. The maximum score obtainable from the SNOT-22 questionnaire was one hundred and ten (110) and the minimum score was zero (0). A low/mild symptom severity equated to a SNOT22 score of 0 to 26 (mild disease), an intermediate/moderate severity score to 27 to 58 (moderate disease) and high/severe symptom severity score equated to 59 and above (severe disease) [14]. After completing the SNOT-22 questionnaire, a second questionnaire (VAS) was handed to these patients. Prior to this, each patient was asked how they perceived their general health and overall sino-nasal symptoms in particular in the last two weeks before presentation. They were instructed to rate the severity of their overall sino-nasal symptoms on a scale of 1 to 10 (The visual analogue scale (VAS) was also deployed to determine the overall symptoms severity score and grade the disease). VAS is calibrated from 0 to 10 where 0 means not troublesome and 10 means most troublesome. A VAS score of 0 to 3 was regarded as a mild disease, a score of 4 to 6 was regarded as moderate disease and a score of 7 to 10 was regarded as a severe disease [16]. Data obtained from both questionnaires were then subjected to a correlation test. The correlation between SNOT-22 and VAS scores was done using Spearman correlation test. Statistical significance was inferred at $\mathrm{p}<0.05$.

\section{Results}

Fifty-two adult patients with CRS were recruited into this study, 23 (44.2\%) males and $29(55.8 \%)$ females, giving a male to female ratio of $1: 1.26$. Their ages ranged from $19-$ 91 years (mean 45.0 \pm 5.5 ) as shown in Figure 1.

Mean symptom severity score using SNOT-22.

Postnasal discharge (dripping at the back of the nose) had the highest severity score among patients at $3.04 \pm 0.14$. Dizziness was the symptom with the least mean severity score at $0.71 \pm 0.31$.

The rhinological symptom domain had the highest severity score distribution and mean total score $(2.39 \pm 0.44)$ followed by the psychological symptom domain (1.42 \pm $0.43)$. The symptom domain titled "others" had the least mean severity score of $1.06 \pm 0.40$ as shown in table 1 . 


\section{Age Distribution of Patients}

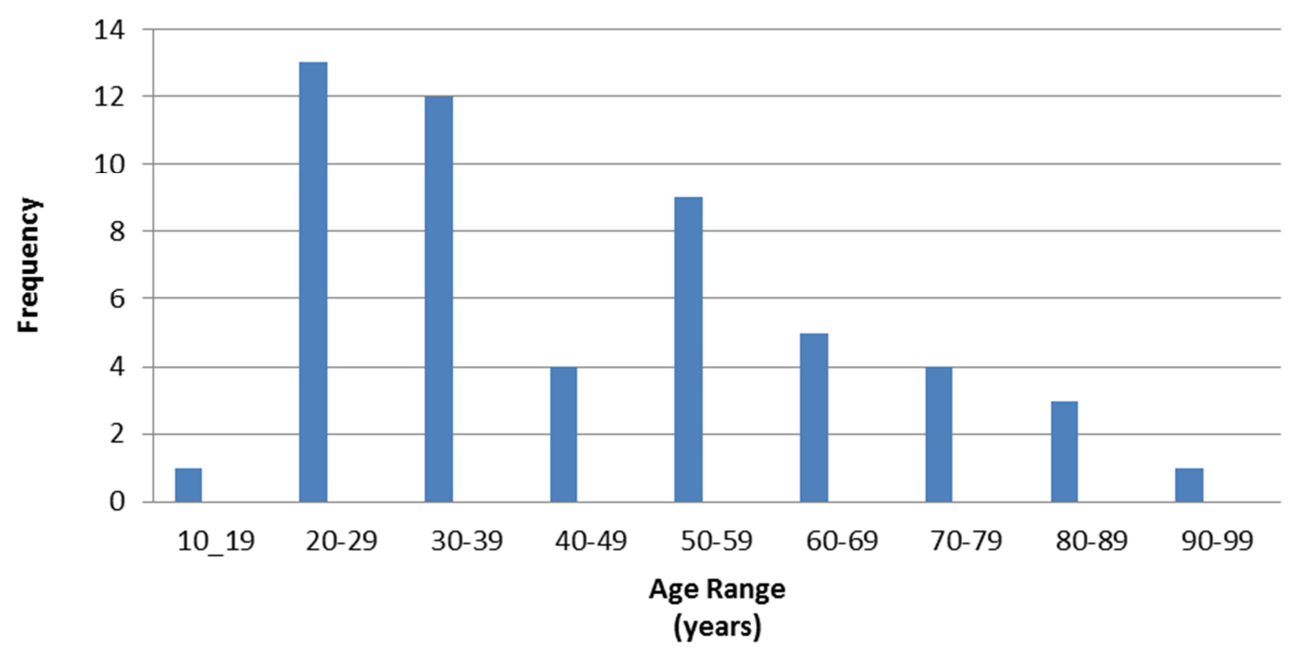

Figure 1. Age distribution of patients.

\section{Distribution of SNOT-22 and VAS scores}

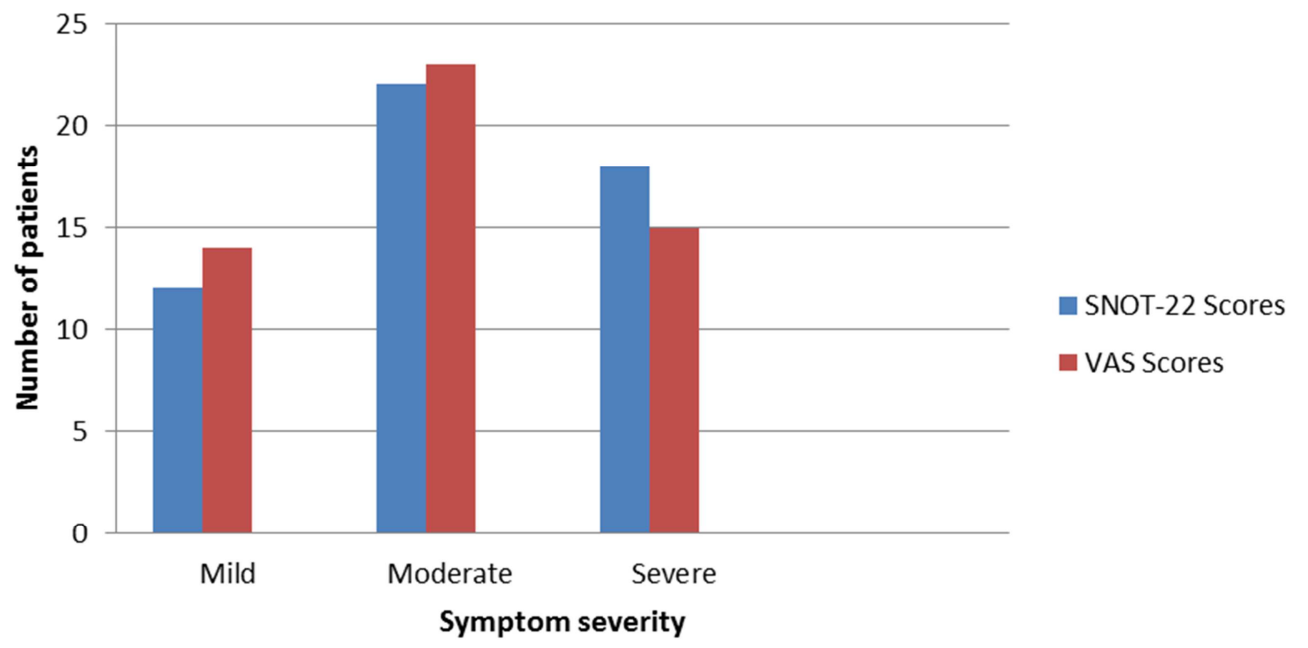

Figure 2. Distribution of SNOT-22 and VAS scores.

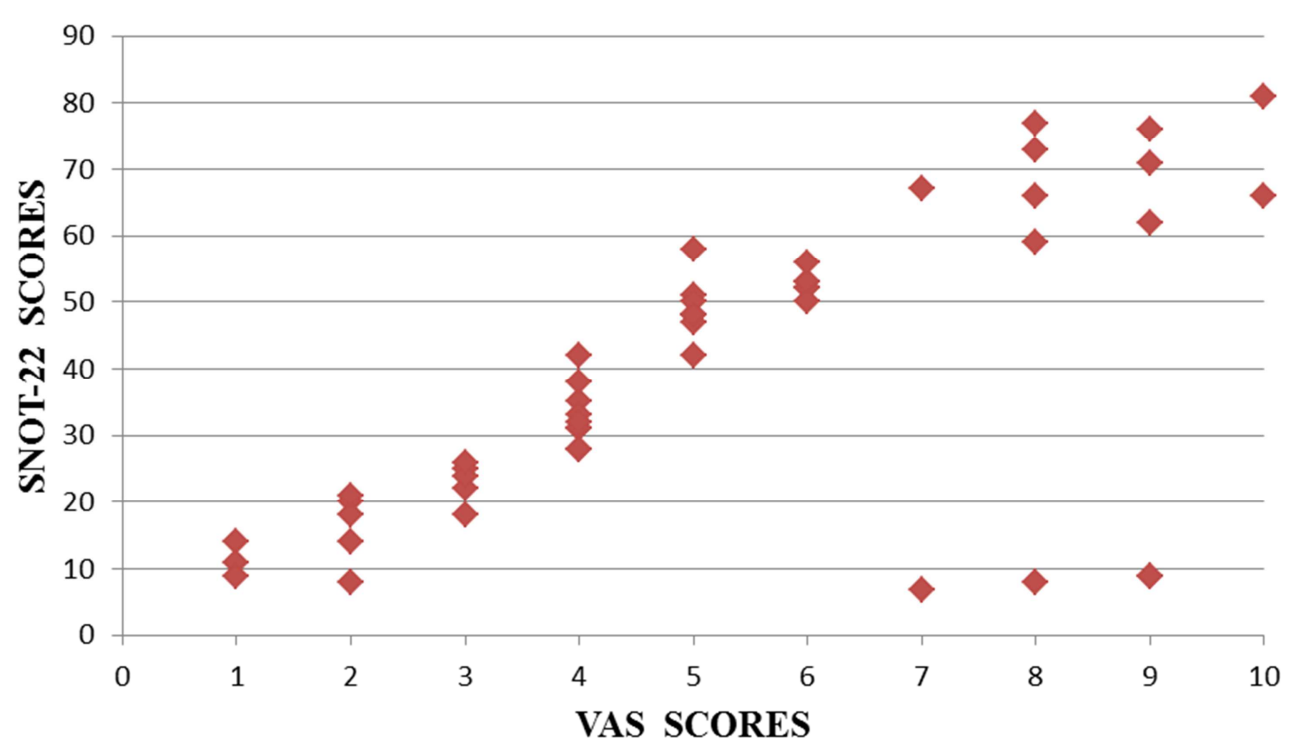

Figure 3. Scatter plot of SNOT-22 scores and VAS scores. 
Table 1. Mean symptom severity score of CRS (Peak score $=5)$.

\begin{tabular}{|c|c|c|c|c|}
\hline Symptom Domain & Sino-Nasal Outcome test & Number of patient & Mean severity score & STD severity score \\
\hline \multirow{7}{*}{ Rhinological Symptoms } & Need to blow nose & 42 & $2.17 \pm .0 .41$ & 1.52 \\
\hline & Sneezing & 41 & $2.21 \pm 0.43$ & 1.59 \\
\hline & Runny Nose & 41 & $2.46 \pm 0.45$ & 1.66 \\
\hline & Post nasal discharge (dripping at back of nose) & 46 & $3.04 \pm 0.41$ & 1.53 \\
\hline & Thick nasal discharge & 41 & $2.29 \pm 0.40$ & 1.65 \\
\hline & Sense of taste/smell & 30 & $1.67 \pm 0.49$ & 1.81 \\
\hline & Blockage /congestion of nose & 45 & $2.88 \pm 0.49$ & 1.79 \\
\hline \multirow[t]{2}{*}{ Total } & & & $2.39 \pm 0.44$ & 1.62 \\
\hline & Ear fullness & 31 & $1.15 \pm 0.32$ & 1.19 \\
\hline \multirow{3}{*}{ Ear/facial symptoms } & Dizziness & 18 & $.71 \pm 0.31$ & 1.14 \\
\hline & Ear pain/pressure & 25 & $.88 \pm 0.64$ & 1.20 \\
\hline & Facial pain/pressure & 35 & $2.13 \pm 0.48$ & 1.77 \\
\hline \multirow[t]{2}{*}{ Total } & & & $1.21 \pm 0.44$ & 1.32 \\
\hline & Difficulty falling asleep & 31 & $2.00 \pm 0.52$ & 1.92 \\
\hline \multirow{3}{*}{ Sleep symptoms } & Waking up at night & 35 & $1.98 \pm 0.46$ & 1.67 \\
\hline & Lack of a good night's sleep & 31 & $1.69 \pm 0.45$ & 1.66 \\
\hline & Waking up tired & 32 & $1.79 \pm 0.47$ & 1.75 \\
\hline \multirow[t]{3}{*}{ Total } & & & $1.24 \pm 0.48$ & 1.75 \\
\hline & Fatigue during the day & 31 & $1.42 \pm 0.40$ & 1.47 \\
\hline & Reduced productivity & 28 & $1.40 \pm 0.42$ & 1.58 \\
\hline \multirow{4}{*}{ Psychological symptoms } & Reduced concentration & 25 & $1.23 \pm 0.41$ & 1.52 \\
\hline & Frustrated /restless/irritable & 28 & $1.40 \pm 0.42$ & 1.55 \\
\hline & Sad & 30 & $1.56 \pm 0.45$ & 1.66 \\
\hline & Embarrassed & 28 & $1.52 \pm 0.45$ & 1.69 \\
\hline Total & & & $1.42 \pm 0.43$ & 1.58 \\
\hline Others & Cough & 24 & $1.06 \pm 0.40$ & 1.49 \\
\hline
\end{tabular}

Distribution of SNOT-22 and VAS scores.

Descriptive statistics of total SNOT-22 scores.

The mean total SNOT-22 of the patients in this study was 38.67 with standard deviation of 17.20 . The median was 41.50 , mode was 42.00, minimum score was 4.0 and maximum score was 76.0. The total SNOT-22 score was not skewed but approximately normally distributed.

A moderate/intermediate severity of CRS was the most prevalent finding among patients administered both questionnaires as shown in figures 2 and 3.
Correlation between SNOT-22 scores and VAS.

There was a significant positive correlation between a high/severe SNOT-22 score and severe VAS scores $(\mathrm{r}=$ $+0.497, \mathrm{p}<0.05$ ), a significant positive correlation between intermediate SNOT-22 scores and moderate VAS scores ( $\mathrm{r}$ $=+0.392, \mathrm{p}<0.05)$ and an insignificant positive correlation between a low SNOT-22 score and mild VAS scores $(\mathrm{r}=$ $+0.285, \mathrm{p}>0.05$ ) as shown in table 2 .

Table 2. Correlation between Overall SNOT-22 and VAS scores.

\begin{tabular}{lll}
\hline Variables correlated & Correlation coefficient $(\mathbf{r})$ & P-value \\
\hline Low symptom scores: SNOT-22 & +0.285 & 0.118 \\
VAS & & \\
moderate symptom scores: SNOT-22 & +0.392 & 0.021 \\
VAS & & \\
High symptom scores: SNOT-22 & +0.497 & 0.008 \\
VAS & \\
\hline
\end{tabular}

\section{Discussion}

Chronic rhinosinusitis (CRS) is a common medical condition of multi-factorial origin that can severely affect the quality of life of individuals who suffer from it. Not all the symptoms of CRS can be precisely described by patients; hence the need arises for a quantifiable, very simple and easily admissible instrument such as VAS; an instrument most commonly used for pain.

There was a slight female preponderance for CRS in our study and the mean age of patients was 45 years, signifying that the disease is more common in middle aged adults. This is in consonance with reports from a similar work by da 
Lilly-Tariah et al [18] but differs from that of Sogebi et al [19] who reported that CRS was more common in the pediatric age group and in young adults with no sex predilection. Our study found the most severe symptoms of CRS in order of decreasing mean severity scores were postnasal dripping (3.04 \pm 0.41$)$, nasal blockage/congestion $(2.88 \pm 0.49)$ and runny nose $(2.46 \pm 0.45)$. This is similar to what was reported by Bhattacharyya et al [20], differing slightly from another contemporary work by Abdalla et al [21] where nasal blockage/congestion, altered taste/smell and need to blow nose were reported as the most severe symptoms of CRS in that order.

The distribution of symptom severity score among the five major symptom domains in this study showed that the rhinological symptom domain had the highest severity scores followed by the psychological symptom domain. However Bhattacharyya et al [22] reported a different outcome, with rhinological symptom domain having the highest severity scores followed by the facial symptom domain. His work was carried out on a population being evaluated for potential chronic rhinosinusitis which also included patients with acute rhinosinusitis. This perhaps explains the difference in outcome.

According to VAS classification of disease severity more than half of the patients in this study adjudged their disease to be moderate or severe. This finding is in consonance with reports from a similar local study by Olowosusi et al [23] where $60.5 \%$ of patients adjudged their disease to be moderate or severe. Although the measure of disease severity using VAS is subjective and open to individual variability, it does provides a quick and simple understanding of an individual's overall disease condition as it affects functionality and also helps to evaluate response to therapy.

This study found a positive correlation between SNOT-22 and VAS scoring of disease severity in moderate and severe diseases and an insignificant correlation between these two instruments in mild disease. This finding demonstrates that a simple, easily administered questionnaire like VAS can achieve the same result as a relatively more complex questionnaire like snot-22. Hence VAS can be used as a guide to symptom severity in the pre-treatment patient.

\section{Conclusion}

VAS can be used as a guide to symptom severity in the pre-treatment patients with CRS, especially in high and moderate severity/ disease where there is a moderate to strong correlation between these two instruments. Though further research on this subject using a larger sample size is advocated for, VAS can be employed to estimate symptom/ disease severity in areas that presents low literacy and language barriers as challenges.

\section{List of Abbreviations}

$\begin{array}{ll}\text { CRS } & \text { Chronic rhinosinusitis } \\ \text { SNOT-22 } & \text { Sinonasal outcome test 22 } \\ \text { VAS } & \text { Visual analogue score } \\ \text { OMC } & \text { Osteomeatal complex }\end{array}$

\section{Declarations}

Ethical approval: Ethical clearance was sought and obtained for this work from the hospital Ethics review committee (Obafemi Awolowo University Teaching Hospital Complex IRB/IEC/0004553, Protocol number: ERC/2013/11/10) after which a written informed consent was obtained from individual participants in this study.

Availability of data and materials: Data and materials used for this work is readily available and can be found in the result section of this manuscript. The authors will gladly provide any further data required during evaluation of this work at any time.

\section{Competing Interests}

The authors declare that they have no competing interests.

\section{Funding}

No funding from institutions, bodies or any organization was received for this study.

\section{Appendix}

\section{Appendix I}

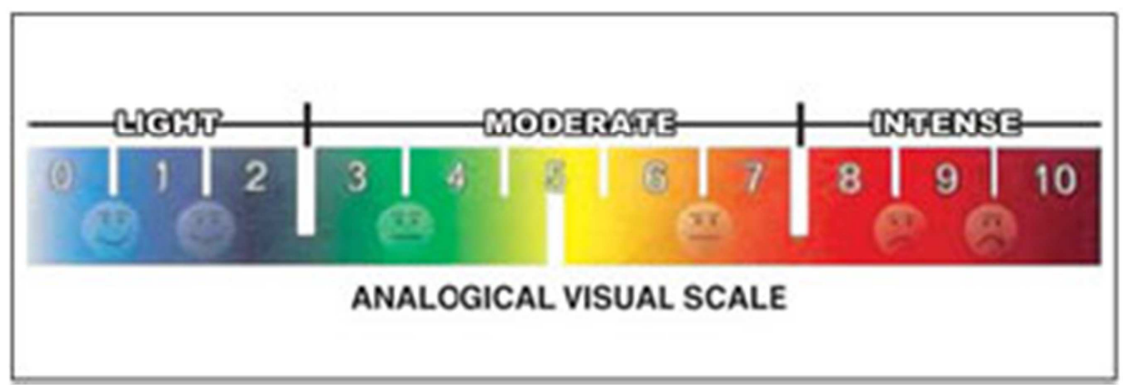




\section{Appendix II}

SNOT -22 QUESTIONNAIRE.

\begin{tabular}{|c|c|c|c|c|c|c|}
\hline $\begin{array}{l}\text { A: Considering how severe the } \\
\text { problem is when you experience } \\
\text { it and how frequently it } \\
\text { happens, please rate each item } \\
\text { below on how 'bad' it is by } \\
\text { circling the number that } \\
\text { corresponds with how you feel } \\
\text { using this scale } \rightarrow\end{array}$ & $\begin{array}{c}\text { No } \\
\text { problem }\end{array}$ & \begin{tabular}{|l|} 
Very mild \\
problem
\end{tabular} & $\begin{array}{l}\text { Mild or } \\
\text { slight } \\
\text { problem }\end{array}$ & $\begin{array}{l}\text { Moderate } \\
\text { problem }\end{array}$ & $\begin{array}{c}\text { Severe } \\
\text { problem }\end{array}$ & $\begin{array}{l}\text { Problem } \\
\text { as bad as } \\
\text { it can be }\end{array}$ \\
\hline 1. Need to blow nose & 0 & 1 & 2 & 3 & 4 & 5 \\
\hline 2. $\quad$ Sneezing & 0 & 1 & 2 & 3 & 4 & 5 \\
\hline 3. Runny nose & 0 & 1 & 2 & 3 & 4 & 5 \\
\hline 4. Cough & 0 & 1 & 2 & 3 & 4 & 5 \\
\hline $\begin{array}{l}\text { 5. Post nasal discharge } \\
\text { (dripping at the back of } \\
\text { your nose) }\end{array}$ & 0 & 1 & 2 & 3 & 4 & 5 \\
\hline 6. Thick nasal discharge & 0 & 1 & 2 & 3 & 4 & 5 \\
\hline 7. Ear fullness & 0 & 1 & 2 & 3 & 4 & 5 \\
\hline 8. $\quad$ Dizziness & 0 & 1 & 2 & 3 & 4 & 5 \\
\hline 9. $\quad$ Ear pain & 0 & 1 & 2 & 3 & 4 & 5 \\
\hline 10. Facial pain/pressure & 0 & 1 & 2 & 3 & 4 & 5 \\
\hline 11. Difficulty falling asleep & 0 & 1 & 2 & 3 & 4 & 5 \\
\hline 12. Waking up at night & 0 & 1 & 2 & 3 & 4 & 5 \\
\hline 13. Lack of a good night's sleep & 0 & 1 & 2 & 3 & 4 & 5 \\
\hline 14. Waking up tired & 0 & 1 & 2 & 3 & 4 & 5 \\
\hline 15. Fatigue & 0 & 1 & 2 & 3 & 4 & 5 \\
\hline 16. Reduced productivity & 0 & 1 & 2 & 3 & 4 & 5 \\
\hline 17. Reduced concentration & 0 & 1 & 2 & 3 & 4 & 5 \\
\hline $\begin{array}{l}\text { 18. Frustrated/restless/ } \\
\text { irritable }\end{array}$ & 0 & 1 & 2 & 3 & 4 & 5 \\
\hline 19. Sad & 0 & 1 & 2 & 3 & 4 & 5 \\
\hline 20. Embarrassed & 0 & 1 & 2 & 3 & 4 & 5 \\
\hline 21. Sense of taste/smell & 0 & 1 & 2 & 3 & 4 & 5 \\
\hline $\begin{array}{l}\text { 22. } \begin{array}{l}\text { Blockage/congestion of } \\
\text { nose }\end{array}\end{array}$ & 0 & 1 & 2 & 3 & 4 & 5 \\
\hline
\end{tabular}

\section{References}

[1] Fokkens, WJ., Lund, VJ., Mullol J European position paper on Rhinosinusitis and Nasal polyps. Rhinology 2007; 20: 1-136.

[2] Bhattacharyya N. The Economic burden and symptom manifestation of chronic rhinosinusitis. AM J Rhinol 2003; 17: 27-32.

[3] Iseh KR, Makusidi M. Rhinosimisitis: A retrospective analysis of clinical pattern and outcome in north western Nigeria. Annals of African Medicine. 2010, 9: 20-26.

[4] Kolo ES. The role of plain radiographs in the diagnosis of chronic maxillary rhinosinusitis in adults. African Health Sciences 2012; 4: 459-463.

[5] Okafor BC. Otolaryngology in South Eastern Nigeria 11 Pattern of diseases of the nose. Niger Med J 1983; 13: 21-9.

[6] Bhatia PC, Varugese R. Pattern of otolaryngological diseases in Jos Community. Niger Med J 1987, 17: 67-73.

[7] Nwawolo CC. Hazards of Maxillary antral Washout. Nig Postgrad Med J 1997; 4: 123-6.
[8] Ahmad BM, Tahir AA. Rhinosinusitis in North Eastern Nigeria. Clinico Radiologic findings. Niger J Med 2000, 9: 21-3.

[9] Ogunleye AO, Nwaorgu OG, Lasisi AO, Ijaduola GT. Trends of sinusitis in Ibadan. West Afr JMed 1999; 18: 298-302.

[10] Desorosiers M, Evans GA, Keith PK, Wright ED, Kaplan A, Bonchard J. Canadian Clinical practice guidelines for acute and chronic rhinosinusitis. J Otolaryngol Head Neck Surg. 2011; 40: 599-193.

[11] Benninger MS, Ferguson BJ, Hadley JA. Adult Chronic Rhinosinusiis: definition, diagnosis, epidemiology, and pathophysiology. Otolaryngol Head Neck Surg. 2003; 129: 1-32.

[12] Frederick J, Braude AL. Anaerobic infection of the paranasal sinuses. N Engl J Med 1974, 200: 135-9.

[13] Bhattacharyya N. Contemporary assessment of the disease burden of sinusitis. Am J Rhinol Allergy. 2009; 23: 392-395.

[14] Pernilla Sahlstrand-Johnson, Bodil Ohlsson, Christian von Buchwald, Magnus Jannert, Marianne Ahlner- Elmqvist. A multicenter study on quality and absenteeism in patients with CRS reffered for endoscopic surgery. Rhinology 2011; 49: 420-428. 
[15] Drazen Shejbal, Davor Vagic, Sinisa Stevanovic, Elvira Koic, Livije Kalogjera. Impact of post-traumatic stress disorder on sinunasal symptoms and quality of life in patients with chronic rhinosinusitis. Patient Prefer Adherence 2012; 6: 847-852.

[16] Lim M, Lew-Gor S, Darby Y, Brookes N, Scaldding G, Lund VJ. The relationship between subjective assessment instruments in chronic rhinosinusitis. Rhinology. 2007; 45: 144-147.

[17] Morley AD, Sharp HR. A review of sinonasal outcome scoring system which is best? Clinical otolaryngology 2006, 31: 103109.

[18] Da Lilly-Tariah O. B. Pattern of clinical features of simple chronic rhinosinusitis in Port Harcourt. Niger j. clin Pract 2006; 9: 142-6.

[19] O. Sogebi, E. Oyewole. Rhinosinusitis clinical features seen in Sagamu, Nigeria. The Internet journal of otorhinolaryngology2006; 6:2.
[20] Bhattacharyya N. Clinical and Symptom Criteria for the accurate Diagnosis of Chronic Rhinosinusitis. Laryngoscope 2006; 116: 1-22.

[21] Abdalla S, Alreely H, Hopkins C. Prevalence of sinonasal outcome test (SNOT-22) symptoms in patients undergoing surgery for chronic rhinosinusitis in England and Wales National prospective audit. Clin Otolaryngol. 2012; 37: 27682.

[22] Bhattacharyya N. A. Comparison of symptom scores and radiographic staging systems in chronic rhinosinusitis. Am J RHINOL. 2005; 19: 175-9.

[23] O. Z Olowosusi, C. N. Asoegwu, A. T. Olagunju, C. C. Nwawolo. A Cross-sectional evaluation of the correlation between disease severity and quality of life in chronic rhinosinusitis patients in Nigeria. Eur Arch Otorhinolaryngol 2014; 14: 23-25. 